\title{
Numerical Comparisons of Nonlinear Convergence Accelerators*
}

\author{
By David A. Smith** and William F. Ford
}

\begin{abstract}
As part of a continuing program of numerical tests of convergence accelerators, we have compared the iterated Aitken's $\Delta^{2}$ method, Wynn's $\varepsilon$ algorithm, Brezinski's $\theta$ algorithm, and Levin's $u$ transform on a broad range of test problems: linearly convergence alternating, monotone, and irregular-sign series, logarithmically convergent series, power method and Bernoulli method sequences, alternating and monotone asymptotic series, and some perturbation series arising in applications. In each category either the $\varepsilon$ algorithm or the $u$ transform gives the best results of the four methods tested. In some cases differences among methods are slight, and in others they are quite striking.
\end{abstract}

1. Introduction. We reported in [19] on a numerical test program that compared eleven different scalar convergence accelerators on a broad range of infinite series problems in an attempt to find an "across-the-board" accelerator that would handle both linear and logarithmic convergence and both alternating and monotone series or sequences. The best candidates to emerge from that study were Levin's $u$ transform [14] and Brezinski's $\theta$ algorithm [6]. We also provided some theoretical support for that conclusion in terms of the wide range of series summed exactly by these methods and by proving that $\theta_{2}$ and the Levin transforms all accelerate linear convergence. (The other Levin transforms, $t$ and $v$, produced essentially equivalent numerical results to $u$, except that $t$ fails on logarithmic convergence.)

We have continued the test program started with [19] to include monotone linearly convergent series, series with irregular sign patterns (specifically, Fourier series), and sequences whose terms do not depend explicitly on the previous term and/or the index (e.g., Bernoulli's method). Since convergence accelerators are often known to transform divergent series to convergent ones, we have also included in our test program divergent power series, asymptotic series, and a few perturbation series.

The methods considered in this paper are the $u$ and $\theta$ algorithms noted above and the "classical" $\varepsilon$ and iterated $\Delta^{2}$ algorithms [17], [23]. All four methods were programmed in their simplest iterative forms, without the use of singular rules or other special devices. The iterative formulas for Levin's $u$ transform were suggested

Received June 4, 1981.

1980 Mathematics Subject Classification. Primary 65B05, 65-04.

Key words and phrases. Acceleration of convergence, iterated Aitken's $\Delta^{2}, \varepsilon$ algorithm, $\theta$ algorithm, Levin's transforms, linear convergence, logarithmic convergence, power series, Fourier series, power method, Bernoulli's method, asymptotic series, perturbation series, numerical tests.

* Part of this material was presented to the Society for Industrial and Applied Mathematics, October 31, 1978, under the title "Transformation of Divergent Series by Convergence Accelerators."

** Work of this author supported in part by NASA grant NSG 3160. 
by F. Cordellier (private communication). The algorithm itself appeared much earlier in work of Bickley and Miller [4, p. 764]; we are indebted to C. Pegis for providing this reference and many other helpful comments.

In all but one of the test problem categories considered here we demonstrate significant (and sometimes striking) results with some or all of the accelerators. The single exception is the class of monotone asymptotic expansions, for which none of the four methods is significantly different from summing to the smallest term, except that each requires more computation than a simple summation.

The results in the other categories force us to modify the conclusion of [19] somewhat. In many cases, the $\varepsilon$ algorithm turns out to be the most effective of the four methods; in all others (including those studied in [19]), the $u$ algorithm does. We found no case in which $\Delta^{2}$ and $\theta$ were not matched or surpassed by one of $\varepsilon$ and $u$.

In the next three sections we describe our numerical results for slowly convergent series (alternating, monotone, and irregular; linear and logarithmic), linearly convergent sequences (power method and Bernoulli's method), and divergent series (alternating and monotone; power and asymptotic series), respectively. Section 5 considers three examples from the applied literature: radiative transfer reflection coefficient [2], the Goldstein series for drag coefficient of a sphere [20], and the RayleighSchrödinger series for ground state energy of the anharmonic oscillator [3]. A summary of the results is given in Section 6. Details of the test problems that have not already been published elsewhere are provided in an Appendix. Our comments include some improvements of numerical answers available from other sources.

Some of the results in this paper have been distributed previously in preprint form under the titles "Nonlinear Acceleration of Linear Convergence" and "Transformation of Divergent Series by Convergence Accelerators". We are indebted to readers and referees of those papers for many helpful suggestions, most of which have been incorporated in the present work. All of the computations reported here have been done in PL/C double precision on an IBM 370/165 at the Triangle Universities Computation Center with programs written by James Treat. Some preliminary results were obtained on an IBM 360 at NASA Lewis Research Center with programs written in SFTRAN by E. C. Bittner. To assure a common basis for comparison of the methods, those computations were repeated with the $\mathrm{PL} / \mathrm{C}$ programs.

2. Slowly Convergent Series. In this section we report numerical tests of the subject algorithms on alternating series, linearly convergent monotone series, logarithmically convergent series (necessarily monotone eventually), and series with irregular sign patterns (Fourier series). The test problems for alternating series and for logarithmically convergent series are the same as those used in [19], with the tests updated to include the iterated $\Delta^{2}$ algorithm.

Table 1 shows the average numbers of significant digits achieved by partial summation ( $S$ column) and each of the four accelerators $\left(\Delta^{2}, \varepsilon, \theta\right.$, and $u$ columns) as functions of the number $n$ of terms of each series used. The averages are taken over the five problems described in [19, Section 5]. The table shows a clear advantage for the $u$-transform, a virtual tie for second between $\boldsymbol{\theta}$ and $\Delta^{2}$, and somewhat slower (but still significant) acceleration by the $\varepsilon$ algorithm. 
TABLE 1

Average numbers of significant digits from $n$ terms of five alternating series by partial summation $(S)$ and four accelerators

\begin{tabular}{r|rrrrr}
$\mathrm{n}$ & $\mathrm{s}$ & $\Delta^{2}$ & $\varepsilon$ & $\theta$ & $\mathrm{u}$ \\
\hline 1 & 0.5 & 0.5 & 0.5 & 0.5 & 0.5 \\
2 & 0.7 & & & & 1.7 \\
3 & 0.9 & 2.1 & 2.1 & 2.8 & 3.1 \\
4 & 1.0 & & & 3.7 \\
5 & 1.1 & 3.8 & 3.7 & & 5.4 \\
6 & 1.2 & & & & 6.2 \\
7 & 1.2 & 5.7 & 5.2 & 5.6 & 7.6 \\
8 & 1.3 & & & & 9.2 \\
9 & 1.3 & 7.7 & 6.7 & & 10.0 \\
10 & 1.4 & 10.0 & 8.3 & 9.0 & 11.4 \\
11 & 1.4 & 10.0 & & 12.9 \\
12 & 1.5 & 12.3 & 9.8 & 12.3 & 15.0 \\
13 & 1.5 & 12.3 & & 15.0 \\
14 & 1.5 & 14.1 & 11.3 & & 15.0 \\
15 & 1.6 & 14.0 \\
16 & 1.6 & 15.0 & 12.9 & & 15.2 \\
17 & 1.6 & 15.0 \\
18 & 1.6 & & & & 15.0 \\
19 & 1.7 & 15.0 & 14.2 & 15.0 & 15.0 \\
20 & 1.7 & & & & 15.0 \\
21 & 1.7 & 9.1 & 15.0 & & 15.0 \\
22 & 1.7 & & & 15.0 & 15.0
\end{tabular}

The same notation will be used in all subsequent tables, but in the interest of saving space, the tables will be condensed. In deference to the $\theta$ algorithm, which requires three terms of the series for each additional entry, each row of the condensed table will present the best result from three consecutive rows of the full table.

Tables 2 and 3 show the results for two groups of monotone linearly convergent series, described in detail in the Appendix. For the first group (Maclaurin-Laurent series for $\tan x$ and other trigonometric functions), the $\varepsilon$ algorithm is the best accelerator, closely followed by $\Delta^{2}$. The $\theta$ algorithm is somewhat slower, and $u$ is slower still, but still significant. The performance of $u$ on the second group (logarithm, dilogarithm, and other series) is very similar to that on the first group, but the other accelerators lose the advantage shown in the first group and lag slightly behind $u$.

\section{TABLE 2}

Average numbers of significant digits from $n$ terms of eight monotone linearly convergent series (Group I: expansions of trigonometric functions)

\begin{tabular}{c|rrrrr}
$\mathrm{n}$ & $\mathrm{s}$ & $\Delta^{2}$ & $\varepsilon$ & \multicolumn{1}{c}{$\theta$} & \multicolumn{1}{c}{$u$} \\
\hline $2-4$ & 0.8 & 1.2 & 1.2 & 1.2 & 1.1 \\
$5-7$ & 1.4 & 7.7 & 7.4 & 4.5 & 3.6 \\
$8-10$ & 2.1 & 10.1 & 11.2 & 8.2 & 5.7 \\
$11-13$ & 2.7 & 13.0 & 14.9 & 12.4 & 7.5 \\
$14-16$ & 3.4 & 14.8 & 14.7 & 12.9 & 9.6 \\
$17-19$ & 4.0 & 9.1 & 14.1 & 14.1 & 10.2 \\
$20-22$ & 4.7 & 11.2 & 13.3 & 13.6 & 10.3
\end{tabular}




\section{TABLE 3}

Average numbers of significant digits from $n$ terms of ten monotone linearly convergent series (Group II: logarithm, dilogarithm, etc.)

\begin{tabular}{c|rrrrr}
$\mathrm{n}$ & $\mathrm{s}$ & $\Delta^{2}$ & $\varepsilon$ & $\theta$ & $\mathrm{u}$ \\
\hline $2-4$ & 1.5 & 1.7 & 1.7 & 2.1 & 2.5 \\
$5-7$ & 2.4 & 4.7 & 4.0 & 4.5 & 4.8 \\
$8-10$ & 3.1 & 6.2 & 5.2 & 6.0 & 7.0 \\
$11-13$ & 3.9 & 8.2 & 7.4 & 7.6 & 8.8 \\
$14-16$ & 4.6 & 9.0 & 8.4 & 8.5 & 10.0 \\
$17-19$ & 5.3 & 9.8 & 9.9 & 9.5 & 10.5 \\
$20-22$ & 6.1 & 10.2 & 10.4 & 9.6 & 10.6
\end{tabular}

In [19] we reported that $u$ and $\theta$ were capable of significant acceleration of logarithmically convergent series, whereas $\varepsilon$ was not. The latter conclusion applies to $\Delta^{2}$ as well, as is shown in Table 4. The test problems are described in [19, Section 6].

\section{TABLE 4}

Average numbers of significant digits from $n$ terms of eight logarithmically convergent series

\begin{tabular}{c|ccccc}
$\mathrm{n}$ & $\mathrm{s}$ & $\Delta^{2}$ & $\varepsilon$ & $\theta$ & $\mathrm{u}$ \\
\hline $2-4$ & 0.7 & 0.8 & 0.8 & 2.1 & 1.9 \\
$5-7$ & 0.9 & 1.6 & 1.2 & 4.0 & 5.0 \\
$8-10$ & 1.0 & 1.7 & 1.4 & 5.9 & 8.0 \\
$11-13$ & 1.1 & 2.2 & 1.6 & 7.9 & 9.5 \\
$14-16$ & 1.2 & 2.3 & 1.7 & 8.6 & 9.8 \\
$17-19$ & 1.2 & 2.4 & 1.9 & 8.8 & 8.0 \\
$20-22$ & 1.3 & 2.6 & 1.9 & 7.8 & 6.1
\end{tabular}

To test the sensitivity of the accelerators to irregular sign patterns (neither alternating nor monotone), we used two Fourier sine series, one slowly convergent and the other rather rapidly convergent, with five values of the argument for each. (Details of the series are in the Appendix.) The average results for the two groups of series are shown in Tables 5 and 6. Only the $\varepsilon$ algorithm is able to achieve significant acceleration for any of these problems, and, in the more rapidly convergent case (Table 6), the other three methods actually degrade the convergence rate.

\section{TABLE 5}

Average numbers of significant digits from $n$ terms of five slowly convergent series with irregular signs

\begin{tabular}{c|ccccc}
$\mathrm{n}$ & $\mathrm{S}$ & $\Delta^{2}$ & $\varepsilon$ & $\theta$ & $\mathrm{u}$ \\
\hline $2-4$ & 0.8 & 1.1 & 1.1 & 1.3 & 1.5 \\
$5-7$ & 1.0 & 2.2 & 3.8 & 2.1 & 1.9 \\
$8-10$ & 1.1 & 2.8 & 5.1 & 3.1 & 2.4 \\
$11-13$ & 1.6 & 3.4 & 7.0 & 3.2 & 2.3 \\
$14-16$ & 1.6 & 3.1 & 7.7 & 3.2 & 2.5 \\
$17-19$ & 1.5 & 3.4 & 8.7 & 3.1 & 2.5 \\
$20-22$ & 1.6 & 3.4 & 9.6 & 3.1 & 2.6 \\
$23-25$ & 1.6 & 3.6 & 10.9 & 2.8 & 2.0 \\
$26-28$ & 1.7 & 3.5 & 11.4 & 3.1 & 1.6 \\
$29-31$ & 1.6 & 3.5 & 12.0 & 2.7 & 1.5
\end{tabular}


TABLE 6

Average numbers of significant digits from $n$ terms of five rapidly convergent series with irregular signs

\begin{tabular}{c|rrrrr}
$\mathrm{n}$ & $\mathrm{s}$ & $\Delta^{2}$ & $\varepsilon$ & $\theta$ & $\mathrm{u}$ \\
\hline $2-4$ & 1.8 & 1.9 & 1.9 & 2.2 & 2.3 \\
$5-7$ & 3.0 & 4.1 & 5.5 & 3.5 & 3.5 \\
$8-10$ & 4.1 & 4.4 & 7.3 & 4.8 & 4.3 \\
$11-13$ & 5.2 & 5.3 & 10.0 & 5.4 & 5.5 \\
$14-16$ & 6.1 & 6.2 & 11.1 & 5.9 & 5.9 \\
$17-19$ & 7.0 & 6.4 & 13.0 & 6.5 & 6.8 \\
$20-22$ & 8.1 & 6.4 & 13.9 & 6.3 & 7.9 \\
$23-25$ & 9.1 & 7.4 & 14.3 & 7.2 & 8.5 \\
$26-28$ & 10.2 & 7.8 & 14.5 & 7.9 & 8.1 \\
$29-31$ & 10.7 & 8.0 & 14.7 & 7.5 & 8.4
\end{tabular}

It has been suggested to us by several readers of the previous draft of this report that a better way to sum Fourier series is to apply complex versions of the accelerators to complex Fourier series and take real and imaginary parts of the results. We agree with that suggestion, and we plan to conduct future tests of complex versions on a variety of problems of interest. However, our point here is to demonstrate the weakness of all but one of these methods on real series that are not either monotone or alternating, a point that is made rather dramatically by Tables 5 and 6.

3. Linearly Convergent Sequences. In this section we present the results from application of the accelerators to linearly convergent sequences whose terms do not depend explicitly on the index $n$, in contrast to the series considered in the previous section. The sequences were generated by the power method for finding the dominant eigenvalue of a square matrix [16, pp. 474-480], using both symmetric and nonsymmetric matrices, and by the Bernoulli method for finding the dominant root of a polynomial [16, pp. 364-368].

\section{TABLE 7}

Average numbers of significant digits from $n$ terms of seven power method sequences generated by symmetric matrices

\begin{tabular}{c|rrrrr}
$\mathrm{n}$ & $\mathrm{S}$ & $\Delta^{2}$ & $\varepsilon$ & $\theta$ & $\mathrm{u}$ \\
\hline $2-4$ & 2.2 & 1.7 & 1.7 & 2.2 & 2.0 \\
$5-7$ & 3.7 & 6.1 & 6.4 & 4.5 & 4.2 \\
$8-10$ & 5.2 & 9.0 & 8.9 & 7.5 & 6.6 \\
$11-13$ & 6.8 & 11.6 & 12.4 & 10.2 & 8.9 \\
$14-16$ & 8.5 & 12.6 & 12.9 & 12.2 & 10.9 \\
$17-19$ & 9.7 & 10.8 & 13.4 & 12.9 & 12.3 \\
$20-22$ & 10.6 & 8.8 & 12.8 & 13.0 & 13.5 \\
$23-25$ & 11.5 & 10.4 & 12.3 & 12.8 & 13.4 \\
$26-28$ & 12.4 & 8.7 & 11.1 & 13.9 & 13.2
\end{tabular}

The scalar sequences from the power method were generated by taking ratios of first entries of the corresponding vector sequences. The symmetric matrices used were Examples 4.1, 2, 4, 5, 6, 8, 9 of [10], and the nonsymmetric matrices were Examples 5.1, 2, 5, 7, 9 of [10]. We used the starting vector $(1,1, \ldots, 1)^{T}$ in all cases except when this was an eigenvector or nearly so, in which case $(1,0,0, \ldots, 0)^{T}$ was used. The power method results are shown in Table 7 for the symmetric case and 
Table 8 for the nonsymmetric case. In the symmetric case, all four methods achieve noticeable acceleration. Differences among the methods are relatively small, but the preference order is $\varepsilon, \Delta^{2}, \theta, u$. In the nonsymmetric case, the same preference order appears, but the $\varepsilon$ algorithm is significantly better than the other methods.

\section{TABLE 8}

Average numbers of significant digits from $n$ terms of five power method sequences generated by symmetric matrices

\begin{tabular}{c|rrrrr}
$\mathrm{n}$ & $\mathrm{s}$ & $\Delta^{2}$ & $\varepsilon$ & $\theta$ & $\mathrm{u}$ \\
\hline $2-4$ & 1.2 & 0.9 & 0.9 & 1.1 & 1.2 \\
$5-7$ & 1.8 & 3.0 & 3.0 & 2.1 & 2.2 \\
$8-10$ & 2.6 & 6.6 & 4.5 & 4.0 & 3.5 \\
$11-13$ & 3.4 & 8.5 & 7.8 & 5.8 & 5.1 \\
$14-16$ & 4.2 & 9.0 & 9.7 & 7.7 & 6.4 \\
$17-19$ & 4.9 & 9.5 & 11.7 & 8.5 & 7.5 \\
$20-22$ & 5.3 & 7.0 & 11.8 & 8.1 & 8.0 \\
$23-25$ & 6.1 & 9.7 & 12.5 & 9.4 & 7.9 \\
$26-28$ & 6.6 & 10.0 & 13.2 & 9.4 & 7.9
\end{tabular}

One subject of Brezinski's paper [7] is combining the vector version of the $\varepsilon$ algorithm and Aitken's $\Delta^{2}$ with the power method to produce more powerful methods for finding all eigenvalues and eigenvectors of a matrix. We have not incorporated these or any other enhancements of the power method, since our objective is not to compute eigenvalues but to test accelerators on slowly convergent sequences.

Test problems for the Bernoulli method were gleaned from a variety of textbook examples. After eliminating rapidly convergent examples, we were left with five polynomials of degrees 3 and 4 (see the Appendix for details). The pattern of results (see Table 9) is similar to those for the power method, with the same preference order among the four methods.

\section{TABLE 9}

Average numbers of significant digits from $n$ terms of five Bernoulli method sequences

\begin{tabular}{c|ccccc}
$\mathrm{n}$ & $\mathrm{s}$ & $\Delta^{2}$ & $\varepsilon$ & $\theta$ & $\mathrm{u}$ \\
\hline $2-4$ & 1.1 & 1.6 & 1.6 & 1.5 & 1.6 \\
$5-7$ & 1.5 & 2.9 & 3.4 & 3.1 & 2.6 \\
$8-10$ & 1.9 & 3.3 & 4.3 & 3.5 & 3.3 \\
$11-13$ & 2.3 & 5.5 & 6.4 & 4.0 & 4.0 \\
$14-16$ & 2.7 & 6.2 & 7.0 & 3.9 & 4.7 \\
$17-19$ & 3.1 & 6.9 & 7.5 & 6.0 & 5.5 \\
$20-22$ & 3.4 & 3.8 & 7.8 & 6.4 & 5.7 \\
$23-25$ & 3.8 & 4.0 & 8.1 & 6.7 & 6.0 \\
$26-28$ & 4.2 & 4.1 & 8.1 & 6.6 & 6.1
\end{tabular}

4. Transformations of Divergent Series. Sequence accelerators may sometimes be used to transform divergent series to meaningful convergent sequences, for example, to "sum" power series beyond their radius of convergence (analytic continuation), or to extend the useful region of asymptotic series. In this section we consider the effect of applying the four accelerators to alternating and monotone power series and to 
alternating and monotone asymptotic series. In each of the first three cases, the results are quite good for at least one of the methods; in the fourth, there is little improvement over summing the series to its smallest term.

Tables 10 and 11 show the average results for three alternating and six monotone divergent power series, respectively. (Details of the test problems are in the Appendix.) There is no $S$ column in these tables, since partial sums are never close to the "correct" answers, that is, to the values of the analytic continuations of the functions represented by the series. All four methods are capable of achieving highly accurate answers in the alternating case, the $u$ transform somewhat more quickly than the others. In the monotone case, the $u$ transform is useless, and the best results are obtained with $\varepsilon$ and $\Delta^{2}$.

TABLE 10

Average numbers of significant digits from $n$ terms of three alternating divergent power series

\begin{tabular}{c|rrrr}
$\mathrm{n}$ & \multicolumn{1}{|c}{$\Delta^{2}$} & \multicolumn{1}{c}{$\varepsilon$} & \multicolumn{1}{c}{$\theta$} & \multicolumn{1}{c}{$\mathrm{u}$} \\
\hline $2-4$ & 1.3 & 1.3 & 1.8 & 2.6 \\
$5-7$ & 3.8 & 3.4 & 3.8 & 5.8 \\
$8-10$ & 5.3 & 4.4 & 6.3 & 8.6 \\
$11-13$ & 8.9 & 6.4 & 9.1 & 11.0 \\
$14-16$ & 10.7 & 7.5 & 10.1 & 13.1 \\
$17-19$ & 12.5 & 9.5 & 13.3 & 12.9 \\
$20-22$ & 12.9 & 10.5 & 12.9 & 11.7 \\
$23-25$ & 13.1 & 12.7 & 12.7 & 11.3
\end{tabular}

TABLE 11

Average numbers of significant digits from $n$ terms of six monotone divergent power series

\begin{tabular}{c|rrrl}
$\mathrm{n}$ & $\Delta^{2}$ & $\varepsilon$ & $\theta$ & $\mathrm{u}$ \\
\hline $2-4$ & 0.4 & 0.4 & 0.2 & 0.0 \\
$5-7$ & 4.2 & 4.1 & 1.8 & 0.8 \\
$8-10$ & 7.3 & 7.0 & 5.4 & 0.6 \\
$11-13$ & 9.5 & 10.5 & 8.2 & 2.0 \\
$14-16$ & 10.2 & 10.6 & 9.0 & 1.2 \\
$17-19$ & 10.2 & 11.0 & 9.2 & 1.7 \\
$20-22$ & 10.1 & 10.5 & 9.3 & 1.8 \\
$23-25$ & 10.0 & 10.9 & 8.9 & 0.8
\end{tabular}

For our alternating asymptotic expansions, we selected 14 series of the form $\sum(-1)^{n+1} a_{n} x^{-n}$, valid for large $x$, but with values of $x$ small enough to produce wild divergence, so that no useful information is obtained directly from partial sums. (See the Appendix for details of the coefficients and values of $x$.) The problems separated themselves into two groups displaying rather different behavior for $\Delta^{2}$ and $\theta$. (The behavior of $u$ and $\varepsilon$ was consistent across all 14 problems, with $u$ achieving significantly better results.) For the 10 problems in Group I, the dependence of $a_{n}$ on $n$ is factorial in nature, and all four accelerators were able to extract useful information, with $u$ achieving the best results, $\varepsilon$ the worst. The other four problems (Group II) have a factor of $n$ in $a_{n}$ (in addition to factorials), and this appears to interfere with the effectiveness of $\Delta^{2}$ and $\theta$. The results for both groups are shown in Table 12. 


\section{TABLE 12}

Average numbers of significant digits from $n$ terms of ten alternating asymptotic series (Group I) and four exceptional cases (Group II)

\begin{tabular}{|c|c|c|c|c|c|c|c|c|}
\hline $\mathrm{n}$ & $\begin{array}{c}\text { Group } \\
\Delta^{2}\end{array}$ & $\varepsilon$ & $\theta$ & $\mathrm{u}$ & $\Delta^{2}$ & $\begin{array}{c}\text { coup } \\
\varepsilon\end{array}$ & $\theta$ & $\mathrm{u}$ \\
\hline $\begin{array}{c}2-4 \\
5-7 \\
8-10 \\
11-13 \\
14-16 \\
17-19\end{array}$ & $\begin{array}{l}1.3 \\
3.1 \\
4.1 \\
6.4 \\
7.2 \\
7.9\end{array}$ & $\begin{array}{l}1.3 \\
2.4 \\
2.8 \\
3.5 \\
3.8 \\
4.3\end{array}$ & $\begin{array}{l}1.9 \\
3.5 \\
5.4 \\
6.6 \\
6.8 \\
8.0\end{array}$ & $\begin{array}{l}2.4 \\
4.5 \\
6.0 \\
7.7 \\
8.8 \\
9.2\end{array}$ & $\begin{array}{l}0.3 \\
1.2 \\
1.4 \\
1.0 \\
1.2 \\
1.1\end{array}$ & $\begin{array}{l}0.3 \\
0.8 \\
1.2 \\
2.7 \\
3.2 \\
3.4\end{array}$ & $\begin{array}{l}0.5 \\
0.5 \\
1.4 \\
0.8 \\
1.5 \\
1.2\end{array}$ & $\begin{array}{l}1.1 \\
1.6 \\
3.2 \\
5.1 \\
7.5 \\
6.8\end{array}$ \\
\hline
\end{tabular}

The test group of monotone asymptotic series consisted of 8 series of the form $\sum a_{n} x^{-n}$ with coefficients scaled so that $x=25,50$, and 100 would represent "small", "medium", and "large" $x$, respectively, for purposes of summing the series to their smallest terms. With small $x$, this produces only a few significant digits of the "answers" represented by the series; with medium $x$, at least eight significant digits; and with large $x$, almost machine accuracy. (Further details of the test problems are in the Appendix.)

The results for these problems, shown in Table 13, can be summarized quite simply. All four accelerators are capable of achieving accuracy comparable to, but not superior to, partial summation. All tended to extract this information from slightly fewer terms of the series, but not enough fewer to warrant the extra computation. The partial sums of course tend monotonically toward their closest approach to the answer before diverging, but that was not always the case with the accelerated sequences. In contrast to the alternating case, the "useful region" for these asymptotic expansions was not extended noticeably by any of the accelerators.

\section{Applications.}

(a) Reflection Coefficients in Radiative Transfer. Bellman and Kalaba [2] have considered the following problem: Suppose parallel rays of radiation are incident upon a plane-parallel slab of thickness $x$ which absorbs radiation and scatters it isotropically. For input and output angles at $60^{\circ}$ to normal they calculated the reflection coefficient at thicknesses from 0 to 1.2 mean free paths in steps of 0.02 . From this slowly increasing sequence of 60 terms they wanted to determine the limit as $x \rightarrow \infty$, i.e., the reflection coefficient for the infinite thickness case, which they knew from other considerations to be 0.272389 .

Their approach to the problem was to apply Aitken's $\Delta^{2}$ process to the known sequence and then do it again to the resulting sequence. From this they concluded: "We see that we may predict a limiting value of about 0.27 which is quite accurate enough for many purposes." In fact, it is difficult to see how this conclusion could be drawn from their data if the answer were not already known. Both $\Delta^{2}$ sequences are initially decreasing and overshoot the limit on their way to a broad, flat minimum at about 0.256 . The first application then rises steadily, but very slowly, to 0.266 , which is suggestive of the desired result, if there were any way to know that it does not rise to 0.28 or beyond. The second application, from which the conclusion 


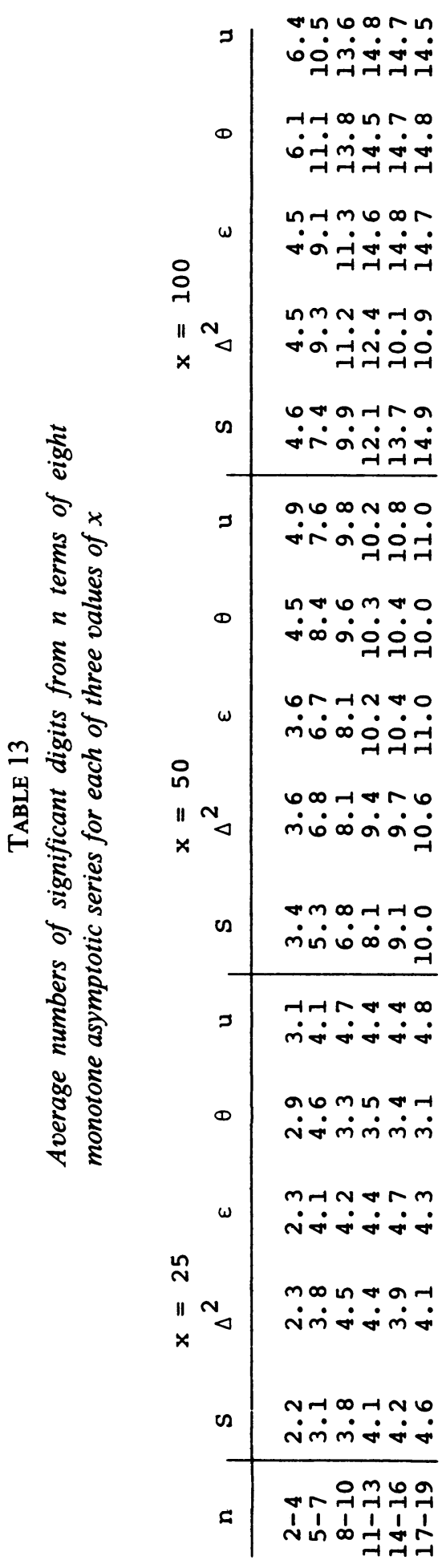


is drawn, produces a sequence that is quite unstable after about the 30th step. While it has an occasional value thereafter in the vicinity of 0.27 , it has many others nowhere near that value.

In contrast to this situation, application of the $\varepsilon$ algorithm to the original sequence produces a sequence the last 10 terms of which (corresponding to the last 20 terms of the original) agree with the desired answer to $3 \mathrm{SD}$, which is about as much accuracy as can be expected from $8 \mathrm{SD}$ monotone data. The $\theta$ algorithm is convincing only for $2 \mathrm{SD}$ (but still better than $\Delta^{2}$ ), and the $u$ algorithm does not produce any useful information.

(b) The Goldstein Series. The drag coefficient $f(R)$ of a sphere as a function of the Reynolds number $R$ is given by the Goldstein series

$$
f(R)=\frac{3}{R} \sum_{n=0}^{\infty} a_{n} R^{n}
$$

where the first 24 coefficients are given to 8D by Van Dyke [20]. This series (in slightly different notation) was used by Shanks [17] to detect an error in Goldstein's evaluation of the sixth coefficient, $a_{5}$, by matching entries in (what we now call) the $\varepsilon$ table with 3 SD values of $f$ obtained by Goldstein by another method (numerical solution of an infinite system of linear equations). The $\varepsilon$ extrapolation from five terms of the series matched quite well, while that based on six terms did not, suggesting the error, which Shanks corrected.

The radius of convergence, $R_{0}$, of the Goldstein series is determined by a singularity at $-R_{0}=-1.04543$, and the coefficients alternate in sign after the first two. Van Dyke observes that the singularity may be mapped to infinity by an Euler transformation (a linear convergence accelerator) to produce a series valid for large $R$ :

$$
f(R)=\frac{3}{R_{0} x} \sum_{n=0}^{\infty} b_{n} x^{n}, \quad x=\frac{R}{R_{0}+R} .
$$

The first 24 coefficients of this series are also given to 8D in [20]. We have $b_{0}=1$, and all subsequent $b_{n}$ 's are negative. It is from the transformed series that Van Dyke obtains useful information about the values of $f$.

We consider the following questions: Can one obtain values of $f$ by applying other accelerators (in particular, $\varepsilon, \theta, u$; $\Delta^{2}$ was not used in this test) to the Goldstein series? (Note that accuracy of results from the transformed series is limited by the accuracy of $R_{0}$, known only to 6SD. The other accelerators are limited only by the accuracy of the coefficients.) How rapidly does the transformed series converge? Can the transformed series be improved by applying the nonlinear accelerators to it?

The answers to these questions are shown in Table 14. For $R=1$, the Goldstein series is convergent, but too slow to provide any useful information. The $\varepsilon$ and $u$ results are good enough to give a more accurate value for $f(1)$ than can be acquired from Eq. (2): 4.8699299. Since the series in Eq. (2) quickly achieves the maximum possible accuracy (6SD, $S$ column in Table 14), there is not much for the accelerators to do in this case.

The fact that $b_{0}$ is quite different from all subsequent $b_{n}$ 's (opposite in sign and relatively large) suggests that better results may be obtained from the $u$ transform by 
applying it to all terms except the first, i.e. using the second column of Levin's array [14] rather than the first. This supposition is borne out by the equation (2) columns headed $u$ and $u^{(2)}$, which come from the first and second columns of the Levin array, respectively. The $u$ column shows worse results than the $S$ column, while the $u^{(2)}$ column is at least a slight improvement.

For two of the three divergent cases, we see that the $\varepsilon$ algorithm gives slightly faster (but not more accurate) results than the Euler transform. The transformed series may also be accelerated slightly, but not made more accurate, by subsequent application of $\varepsilon$ or $u^{(2)}$. The $\theta$ algorithm and direct application of the $u$ transform seem not to be very useful for this problem.

TABLE 14

Significant digits in drag coefficients $f(R)$ as a function of number $n$ of terms of Goldstein's series

\begin{tabular}{|c|c|c|c|c|c|c|c|c|c|}
\hline \multirow[b]{2}{*}{$\mathbf{R}$} & \multirow[b]{2}{*}{$\mathrm{n}$} & \multicolumn{3}{|c|}{ Equation (1) } & \multicolumn{3}{|c|}{ Equation (2) } & \multirow[b]{2}{*}{$\mathrm{u}$} & \multirow[b]{2}{*}{$u^{(2)}$} \\
\hline & & $\varepsilon$ & $\theta$ & $\mathrm{u}$ & S & $\varepsilon$ & $\theta$ & & \\
\hline 1 & $\begin{array}{l}5-7 \\
8-10 \\
11-13 \\
14-16 \\
17-19 \\
20-22\end{array}$ & $\begin{array}{l}3 \\
3 \\
6 \\
7 \\
8 \\
8\end{array}$ & $\begin{array}{l}1 \\
4 \\
4 \\
3 \\
6 \\
6\end{array}$ & $\begin{array}{l}2 \\
5 \\
7 \\
7 \\
8 \\
8\end{array}$ & $\begin{array}{l}3 \\
5 \\
6 \\
6 \\
6 \\
6\end{array}$ & $\begin{array}{l}2 \\
4 \\
6 \\
6 \\
6 \\
6\end{array}$ & $\begin{array}{l}2 \\
2 \\
5 \\
5 \\
5 \\
6\end{array}$ & $\begin{array}{l}2 \\
3 \\
5 \\
6 \\
6 \\
6\end{array}$ & $\begin{array}{l}3 \\
6 \\
6 \\
6 \\
6 \\
6\end{array}$ \\
\hline 5 & $\begin{array}{l}5-7 \\
8-10 \\
11-13 \\
14-16 \\
17-19 \\
20-22\end{array}$ & $\begin{array}{l}1 \\
1 \\
2 \\
4 \\
4 \\
4\end{array}$ & $\begin{array}{l}1 \\
2 \\
1 \\
2 \\
0 \\
1\end{array}$ & $\begin{array}{l}1 \\
2 \\
3 \\
4 \\
3 \\
2\end{array}$ & $\begin{array}{l}2 \\
2 \\
3 \\
3 \\
3 \\
4\end{array}$ & $\begin{array}{l}1 \\
3 \\
4 \\
4 \\
5 \\
5\end{array}$ & $\begin{array}{l}1 \\
1 \\
1 \\
2 \\
1 \\
3\end{array}$ & $\begin{array}{l}1 \\
1 \\
3 \\
2 \\
3 \\
3\end{array}$ & $\begin{array}{l}1 \\
3 \\
3 \\
4 \\
4 \\
4\end{array}$ \\
\hline 10 & $\begin{array}{l}5-7 \\
8-10 \\
11-13 \\
14-16 \\
17-19 \\
20-22\end{array}$ & $\begin{array}{l}1 \\
1 \\
2 \\
3 \\
3 \\
2\end{array}$ & $\begin{array}{l}0 \\
1 \\
1 \\
1 \\
2 \\
1\end{array}$ & $\begin{array}{l}1 \\
2 \\
2 \\
2 \\
2 \\
0\end{array}$ & $\begin{array}{l}1 \\
2 \\
2 \\
2 \\
2 \\
3\end{array}$ & $\begin{array}{l}1 \\
2 \\
3 \\
3 \\
4 \\
4\end{array}$ & $\begin{array}{l}1 \\
1 \\
1 \\
1 \\
1 \\
3\end{array}$ & $\begin{array}{l}1 \\
1 \\
2 \\
2 \\
2 \\
2\end{array}$ & $\begin{array}{l}0 \\
2 \\
2 \\
3 \\
4 \\
3\end{array}$ \\
\hline 25 & $\begin{array}{l}5-7 \\
8-10 \\
11-13 \\
14-16 \\
17-19 \\
20-22\end{array}$ & $\begin{array}{l}1 \\
1 \\
1 \\
2 \\
2 \\
1\end{array}$ & $\begin{array}{l}0 \\
1 \\
0 \\
0 \\
0 \\
0\end{array}$ & $\begin{array}{l}0 \\
1 \\
2 \\
1 \\
2 \\
0\end{array}$ & $\begin{array}{l}1 \\
1 \\
2 \\
2 \\
2 \\
2\end{array}$ & $\begin{array}{l}1 \\
2 \\
2 \\
2 \\
3 \\
3\end{array}$ & $\begin{array}{l}1 \\
1 \\
1 \\
0 \\
1 \\
0\end{array}$ & $\begin{array}{l}1 \\
1 \\
2 \\
2 \\
2 \\
2\end{array}$ & $\begin{array}{l}0 \\
2 \\
3 \\
3 \\
3 \\
2\end{array}$ \\
\hline
\end{tabular}

(c) The Rayleigh-Schrödinger Series. Bender and $\mathrm{Wu}$ [3] have computed the first 75 terms of the Rayleigh-Schrödinger series for the ground state energy $E(1, \beta)$ of the anharmonic oscillator. Simon [18] has reported extensive computations with this series using the diagonal of the Padé table (which is theoretically equivalent to using the $\varepsilon$ algorithm [17], [23]), and Graffi, et al. [9] have obtained more accurate results by a Borel-Padé summation technique. In particular, Tables 2 and 3 in [9] give the most accurate values available for $E(1, \beta), \beta=0.1(0.1) 1.0$ and $\beta=1(1) 14$. We have computed the effect of accelerating the series by $\varepsilon, \theta$, and $u$ for $\beta=0.1,0.5,1,5$, and 10 , to compare the use of these simpler accelerators to the Borel-Padé summations. 
The results of these computations are shown in Table 15 for small $\beta$ and in Table 16 for intermediate $\beta$. The results for $\varepsilon$ are similar, but not identical, to those given by Simon [18] for Padé approximants. Different methods of evaluation of the same quantities can produce noticeably different numerical results, especially when transforming rapidly divergent series. The $\theta$ and $u$ results are better than those from $\varepsilon$ (or Padé) in every case, and they are nearly as good as the Borel-Padé results in [9]. Graffi, et al., give rate of convergence data only for $\beta=0.1$ and 1.0 (their Table 1).

For $\beta=5$ and 10 , Table 16 shows the digits of $E(1, \beta)$ that one might believe correct if only a single accelerator were used, and the range of $n$ over which these answers are constant. These results show that the $\varepsilon$ computation would be somewhat misleading, but $\theta$ and $u$ would not be.

TABLE 15

Significant digits of $E(1, \beta)$ as a function of number $n$ of terms in the Rayleigh-Schrödinger series. Data in the Borel-Padé columns (B) are taken from Tables 1 and 2 in [9].

$$
\beta=0.1 \quad B=0.5 \quad B=1.0
$$

\begin{tabular}{c|rrrr|rrrrrrrr}
$\mathrm{n}$ & $\varepsilon$ & $\theta$ & $\mathrm{u}$ & $\mathrm{B}$ & $\varepsilon$ & $\theta$ & $\mathrm{u}$ & $\mathrm{B}$ & $\varepsilon$ & $\theta$ & $\mathrm{u}$ & $\mathrm{B}$ \\
\hline $1-5$ & 3 & 1 & 3 & 6 & 2 & 1 & 2 & & 1 & 1 & 1 & 2 \\
$6-10$ & 6 & 7 & 8 & 8 & 3 & 4 & 5 & & 2 & 3 & 6 & 4 \\
$11-15$ & 8 & 9 & 10 & 11 & 4 & 6 & 6 & & 3 & 4 & 5 & 5 \\
$16-20$ & 10 & 11 & 12 & 15 & 5 & 7 & 6 & & 3 & 6 & 5 & 7 \\
$21-25$ & 12 & 11 & 14 & 15 & 6 & 7 & 8 & 9 & 4 & 6 & 6 & 7 \\
$26-30$ & 13 & 9 & 14 & & 6 & 7 & 6 & & 5 & 5 & 3 & \\
$31-35$ & 14 & 14 & 12 & & 7 & 7 & 3 & & 5 & 5 & & \\
$36-40$ & 15 & 14 & 10 & & 7 & 7 & & & 5 & 5 &
\end{tabular}

TABLE 16

Best estimates of $E(1, \beta)$ for intermediate $\beta$. Columns headed " $n$ " indicate numbers of terms of the series for which each method gave the answer shown. Data for the Borel method are from Table 3 of [9]

\begin{tabular}{|c|c|c|c|c|c|c|c|c|}
\hline$B$ & $\varepsilon$ & $\mathrm{n}$ & $\theta$ & $\mathrm{n}$ & $\mathrm{u}$ & $\mathrm{n}$ & Borel & $\mathrm{n}$ \\
\hline $\begin{array}{r}5 \\
10\end{array}$ & $\begin{array}{l}1.0 \\
2.3\end{array}$ & $\begin{array}{l}39-41 \\
31-33\end{array}$ & $\begin{array}{l}2.018 \\
2.45\end{array}$ & $\begin{array}{l}19-28 \\
22-31\end{array}$ & $\begin{array}{l}2.02 \\
2.45\end{array}$ & $\begin{array}{l}10-14 \\
11-13\end{array}$ & $\begin{array}{l}2.017 \\
2.440\end{array}$ & $\begin{array}{l}?-21 \\
?-21\end{array}$ \\
\hline
\end{tabular}

The radius of convergence of the Rayleigh-Schrödinger series is zero, that is, all of the "accelerated" results are obtained from transformations of divergent series. However, this series shares with the Goldstein series the property of having two terms of the same sign before becoming alternating. One might suppose that, as in the case of the Euler-transformed Goldstein series (2), better results would be obtained from the second column of the $u$ array or the second diagonal of the $\varepsilon$ and $\theta$ tables. However, we found no significant differences in results for either the untransformed Goldstein series (1) or the Rayleigh-Schrödinger series from these deferred applications. We conclude that the $u$ transform, at least, is sensitive to the 
starting point in an "eventually monotone" series, but none of the accelerators displayed this sensitivity for an "eventually alternating" series.

6. Summary and Conclusions. The numerical evidence presented here suggests grouping the types of series and sequences considered into a small number of categories, in each of which a rule of thumb emerges. In the absence of theoretical support, these "rules" can never be more than that, of course.

(a) Alternating Series. The evidence of Tables 1, 10, and 12 suggests that, of the four methods tested, the $u$ algorithm is the best for accelerating alternating series (convergent, divergent, asymptotic), $\theta$ and $\Delta^{2}$ are virtually tied for second best (but with an exception to be noted), and $\varepsilon$ is least effective (though still quite good). Most of the series encountered in practice have coefficients that are factorial in nature, but when this is not the case and the series is divergent (as with Group II in Table 12), $\theta$ and $\Delta^{2}$ are susceptible to failure.

(b) Monotone Linearly Convergent Series, Other Linearly Convergent Sequences (Power and Bernoulli Methods) and Monotone Divergent Series. For the groups represented by Tables 2, 7, 8, 9, and 11 (but not Tables 3 and 13) we find the four methods ranked in decreasing order: $\varepsilon, \Delta^{2}, \theta, u$. The exceptional cases in Table 3 are convergent power series with nonfactorial coefficients; here there is significant acceleration, but the differences among the four methods are not significant. For monotone asymptotic series (Table 13), neither the acceleration nor the differences among methods are significant.

(c) Logarithmic Convergence (Table 4). Only the $u$ and $\theta$ algorithms are capable of significant acceleration of logarithmically convergent series, and $u$ is slightly better than $\theta$.

(d) Irregular Sign Patterns (Tables 5 and 6). The $\varepsilon$ algorithm is the least sensitive of these methods to sign patterns and the only one of the four capable of significant acceleration of all the Fourier series tested.

Appendix: Details of Test Problems: Those test problems that are not already described elsewhere are given in detail here, along with some comments about individual problems and some improvements in published answers.

(a) Fourier Sine Series. The series used to determine the effect of irregular sign patterns (Tables 5 and 6) were, respectively,

$$
\begin{aligned}
& \sum_{n=1}^{\infty} \frac{\sin n x}{n}=(\pi-x) / 2, \quad 0<x<2 \pi ; \\
& \sum_{n=1}^{\infty} \frac{\sin n x}{n 2^{n}}=\tan ^{-1}\left(\frac{\sin x}{2-\cos x}\right), \quad 0<x<2 \pi .
\end{aligned}
$$

In each case we used $x=1(1) 5$.

(b) Power Method. The test problems for Tables 7 and 8 all come from Chapters 4 and 5 of [10] and are listed in Section 3 above. A by-product of our computation is a list of improved answers for some of the examples in [10] as shown in Table A1. Some of these are merely improvements in accuracy, but some are corrections of the final digits reported in [10]. In particular, the results for Examples 4.8 and 4.9 cast some doubt on whether the separation of the two largest eigenvalues is as indicated in [10], or indeed if there is any at all. 
TABLE Al

Corrected answers for dominant eigenvalues of matrix examples in Gregory-Karney [10]

\begin{tabular}{c|c|c} 
Example & Answer in [10] & Corrected Answer \\
\hline 4.4 & 30.28868 & 30.2886853458021 \\
4.5 & 1.67828 & 1.67827983 \\
4.6 & 22.40687532 & 22.4068753075804 \\
4.8 & 16.1427446553 & 16.1427446551220 \\
4.9 & 12.41133643 & 12.4113364117214 \\
5.10 & 1.8390 & 1.8390366545819
\end{tabular}

The power method can produce some (well-known) anomalies. Example 4.3 in [10] is the infamous matrix of Bodewig [5], a 4 by 4 symmetric matrix with small integer entries which requires many hundreds of iterations with the power method before the sequence gets near the dominant eigenvalue $\lambda_{1}$. The second eigenvalue $\lambda_{2}$ is nearly equal in magnitude and opposite in sign, and the starting vector $(1,1,1,1)^{T}$ is nearly orthogonal to the dominant eigenvector. The latter fact causes the sequence to first approach $\lambda_{2}$ [16], and the former drastically slows the eventual convergence to $\lambda_{1}$. One may reasonably ask whether the accelerators can bypass the approach to $\lambda_{2}$ or if they hasten it as well as the approach to $\lambda_{1}$. Our evidence indicates the latter. All four accelerators in fact get closer to $\lambda_{2}$ faster than the power sequence, and $\varepsilon$ actually produces $9 \mathrm{SD}$ of $\lambda_{2}$ from the first 50 terms of the sequence. (The extremely slow convergence of the power sequence on problems of the Bodewig type is easy to avoid; see [16] or [21].)

Example 4.5 of [10] is another for which the first "approach" is to $\lambda_{2}$. The accelerators produced 3 to $5 \mathrm{SD}$ of $\lambda_{2}$ from the first 3 to 19 terms before turning their attention to $\lambda_{1}$. All four achieve $9 \mathrm{SD}$ of $\lambda_{1}$, at $n=31$, while the power sequence is converged to $6 \mathrm{SD}$ at this point. For Example 5.5, the initial approach of the power sequence is to the third largest eigenvalue, $\lambda_{3}=2$, and this is the "answer" found by the accelerators as well.

Several of the test problems had two or more equal or nearly equal dominant roots, which seemed to cause no problems for either the power method or the accelerators. On the other hand, when the power sequence failed to converge due to complex dominant roots [10, Example 5.26], the accelerators also failed to converge to anything.

Example 4.7 of [10] produced some unanticipated difficulties and behavior by the accelerators that does not fit the general pattern at all. The example is a 5 by 5 matrix with small integer entries and reasonably separated positive eigenvalues. (There is a misprint in the original source [15], faithfully reproduced in [10]: The 4,5 -entry of the matrix should be -1 , not 1.) The power sequence quickly approaches $\lambda_{2}(3 \mathrm{SD})$ and then becomes a monotone increasing sequence whose graph is concave upward until about $n=20$, which effectively obscures its limit and apparently confuses the accelerators. After passing the inflection point, the $u$ 
algorithm quickly converges to $6 \mathrm{SD}$ of $\lambda_{1}$, but the other accelerators can achieve no more than 1 SD from 30 terms, worse than $S$ itself. This was the only example we found of a power sequence for which $u$ displayed clear superiority.

The same example has been used by Iguchi [12], [13], and his methods are worth commenting on in this connection. These papers consider ways to overcome initial instability in Aitken's $\Delta^{2}$ process by using a short, finite geometric series instead of an infinite series. In [12], Iguchi makes the choice of which series to use depend on an early estimate of the ultimate ratio of terms, and in [13] he uses the short series initially and then switches to $\Delta^{2}$. By both methods, he appears to get results at least as good as the $u$ transform on Example 4.7 of [10]. However, we have not found any other problems for which either of Iguchi's methods works better than $\varepsilon$, say.

(c) Bernoulli Method. The test polynomials for determination of dominant roots by Bernoulli's method are shown in Table A2. Behavior of individual sequences varied considerably from the averages shown in Table 9. For the first problem, the Bernoulli sequence converged to $11 \mathrm{SD}$ in 30 steps, while all four accelerators produced $15 \mathrm{SD}$ (the maximum possible) from fewer terms. For the second and third problems, only $\varepsilon$ was able to produce more than 1 SD from 30 terms, and it could reach only $4 \mathrm{SD}$ and $3 \mathrm{SD}$ respectively. The behavior of all methods on the other two problems closely resembled the averages. The answers shown in Table A2 for those two problems are more accurate than those given in the original source [11].

\section{TABLE A2}

Polynomials used for Bernoulli method tests

\begin{tabular}{rrrrr|ll|l}
$x^{4}$ & $x^{3}$ & $x^{2}$ & $x$ & 1 & Dominant root & Source \\
\hline & 1 & -5 & -17 & 21 & 7.0 & & {$[16$, p. 366] } \\
1 & -3 & -60 & 150 & 300 & 7.6132307 & {$[16$, p. 393] } \\
& 10 & -21 & -40 & 84 & 2.1 & & {$[16$, p. 393] } \\
128 & -140 & 90 & -20 & 1 & 0.9305681557970 & {$[11, \mathrm{p} .148]$} \\
& -256 & 160 & -32 & 1 & 0.9619697662556 & {$[11, \mathrm{p} .171]$}
\end{tabular}

(d) Divergent Power Series. The series used to obtain Table 10 were the alternating sums of $2^{n} / n, 3^{n} / n$, and $3^{n} / n^{2}$, with respective answers $\ln 3, \ln 4$, and

$$
\int_{1}^{4} \frac{\ln t}{t-1} d t=1.939375420767 \ldots
$$

(See [1, 27.7.1, 2, 5 and Table 27.7].) The monotone series used for Table 11 were the Laurent-Maclaurin expansions of $\tan x$ and $\sec x$ for $x=2$ and 3, and of $\cot x$ and $\csc x$ for $x=5$. (See $[1,4.3 .67-70]$.)

(e) Alternating Asymptotic Expansions. The test problems for Table 12 are shown in Tables A3 and A4. The notation for special functions is that of [1]. Digits shown in parentheses were obtained from four or more consecutive terms of the $u$ transform sequence (and not contradicted by any of the other methods). All other answers were obtained independently from formulas in [1]. 


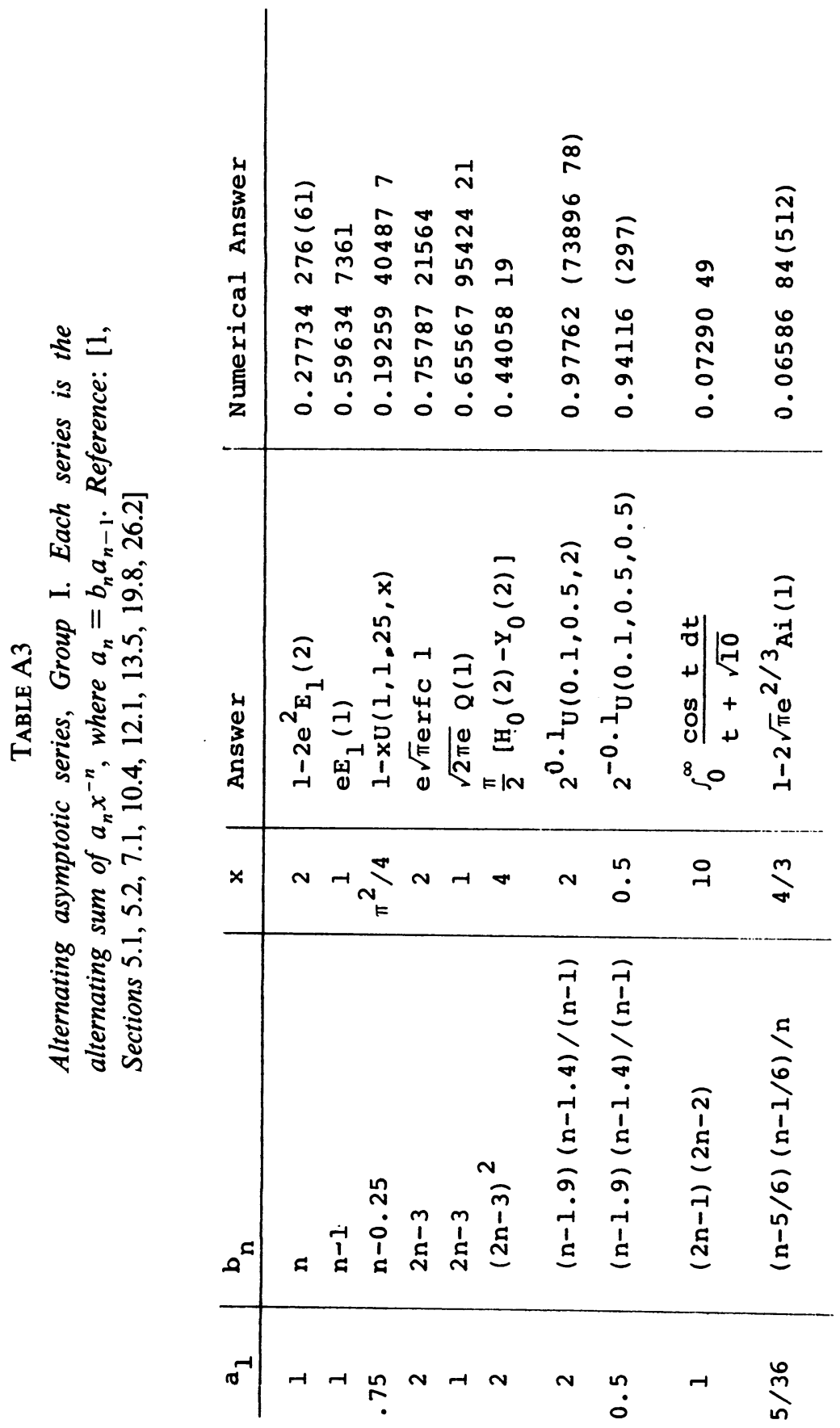




\section{TABLE A4}

Alternating asymptotic series, Group II. Each series is the alternating sum of $a_{n} x^{-n}$. Reference: [1, Sections 13.4, 5, 6]

\begin{tabular}{l|l|l|l}
$a_{n}$ & $x$ & Answer & Numerical Answer \\
\hline$n(n+1) !$ & 2 & $8 U(3,2,2)$ & $0.2187421(3)$ \\
$n(n+1) !$ & 1 & $2 U(3,2,1)$ & 0.21095791 \\
$n(n+1)(n+2) !$ & 2 & $96 U(4,2,2)$ & 0.40616305 \\
$n(n+1)(n+2) !$ & 1 & $12 U(4,2,1)$ & 0.24748431
\end{tabular}

We included among the test problems the sum of $n(2 n-1)(2 n+1) 2^{n}$, which is the value at $x=\sqrt{2}$ of the series expansion of a rational function: $6\left(x^{4}+6 x^{2}+1\right) /\left(1-x^{2}\right)^{4}$. The $\varepsilon$ algorithm is exact on 9 terms of such an expansion [17, Theorem IX], but in fact it gets only 12 correct digits of the right answer, when computed in double precision, due to roundoff. The $u$ transform, which is not exact, gets the answer to $6 \mathrm{SD}$ from 14 terms of the series, and $\theta$ fails to get any correct digits.

\section{TABLE A5}

Monotone asymptotic series. (a) The first six problems are of the form $\sum a_{n} / x^{n}$ and have answers of the form $-P(x)+$ $x Q(x) e^{-x} E i(x)$, where $P$ and $Q$ are the tabulated polynomials.

Reference: [1, Sections 5.1, 10.2, 13.4, 13.5, 13.6]. (b) The other two problems are of the form $\sum a_{n} x^{n-1}$, where $a_{n}=b_{n} a_{n-1}$.

Reference: [1, Formula 9.7.1 and Table 9.8].

(a)

\begin{tabular}{|c|c|c|}
\hline \multirow{2}{*}{$\begin{array}{c}\text { Coefficients } \\
a_{n}\end{array}$} & \multicolumn{2}{|c|}{ Answers } \\
\hline & $\mathrm{P}(\mathrm{x})$ & $Q(x)$ \\
\hline $\mathrm{n} !$ & 1 & 1 \\
\hline$n(n+1) !$ & $x(x-1)$ & $x(x-2)$ \\
\hline$n(n+1)(n+2) !$ & $x^{2}\left(x^{2}-5 x+2\right)$ & $x^{2}\left(x^{2}-6 x+6\right)$ \\
\hline$n^{2}(n+1) !$ & $x\left(x^{2}-4 x+1\right)$ & $x(x-1)(x-4)$ \\
\hline$n^{3}(n+1) !$ & $x\left(x^{3}-8 x^{2}+12 x-1\right)$ & $x\left(x^{3}-9 x^{2}+19 x-8\right)$ \\
\hline$n^{4}(n+1) !$ & $x(x-1)\left(x^{3}-12 x^{2}+31 x-1\right)$ & $x\left(x^{4}-14 x^{3}+55 x^{2}-65 x+16\right)$ \\
\hline
\end{tabular}

(b)

\begin{tabular}{l|l|c}
\multicolumn{2}{l|}{$\begin{array}{l}\text { Coefficients } \\
a_{1}\end{array} b_{n}$ Answers } \\
\hline 1 & $5(2 n-3)^{2} / 8(n-1)$. & $\sqrt{2 \pi x / 5} e^{-x / 5} I_{0}(x / 5)$ \\
-1 & $5(2 n-1)(2 n-5) / 8(n-1)$ & $-\sqrt{2 \pi x / 5} e^{-x / 5} I_{1}(x / 5)$
\end{tabular}


It is well known that analytic representations for "sums" of asymptotic series are not unique. For example, the sum in the third problem of Table A3 can be written

$$
\sum_{n=1}^{\infty} \frac{(-1)^{n+1} 3 \cdot 7 \cdot 11 \cdots(4 n-1)}{\pi^{2 n}},
$$

which is an asymptotic series for

$$
1+\frac{\pi}{2}-\pi \int_{0}^{1} \cos \left(\frac{\pi}{2} t^{2}\right) d t=0.12068 \ldots
$$

[1, 7.3.27 and Table 7.8], but the accelerators all recognized the series as representing a confluent hypergeometric function.

(f) Monotone Asymptotic Expansions. The test problems for Table 13, and their numerical answers, are shown in Tables A5 and A6. For $x=100$ and $x=50$, the numerical answers were obtained from the asymptotic series to the number of digits shown. For $x=25$, the formulas in Table A5 were used. The first six of these involve high loss-of-significance; we computed $25 e^{-25} \operatorname{Ei}(25)=1.0436619362666$, using [1, Formula 5.1.10]. For the other two, Table 9.8 of [1] suffices.

\section{TABLE A6}

Numerical answers for the eight problems in Table A5 for each of three values of $x$

\begin{tabular}{llll|lll|ll}
\multicolumn{1}{c|}{$x=100$} & & \multicolumn{2}{|c|}{$x=50$} & \multicolumn{2}{|c}{$x=25$} \\
\hline 0.01020 & 62527 & 74835 & 7 & 0.02085 & 22777 & 97199 & 0.04366 & 19362 \\
0.02127 & 71933 & 89970 & 0 & 0.04546 & 67132 & 7847 & 0.10561 & 33532 \\
0.13600 & 10469 & 98297 & 0.31205 & 1554 & & 0.86959 & 0 \\
0.02263 & 72038 & 59953 & 0 & 0.05170 & 77443 & 686 & 0.14039 & 6964 \\
0.02553 & 50486 & 38497 & 0.06599 & 48948 & 44 & 0.23717 & 26 \\
0.03190 & 38772 & 20799 & 0.10090 & 5796 & & 0.55749 & 0 \\
1.00643 & 57190 & 3983 & 1.01329 & 0709 & & 1.02874 & 4642 \\
-.98094 & 32134 & 34997 & -.96120 & 73907 & & -.91906 & 31157
\end{tabular}

We discovered by accident an exception to the observation in Section 4 that none of the accelerators extend the "useful region" for monotone asymptotic series. For the second problem of Table A5 with $x=2$ and the fourth problem with $x=1$ or 4 , the $u$ transform gives an exact answer $(-2,2,-4$, respectively) in a small number of steps $\left(4,3,5\right.$, respectively). (The second of these three results is also obtained by $\theta_{2}$ and $\varepsilon_{2}=\Delta^{2}$.) Note that these values of $x$ are the integer roots of the polynomials $Q(x)$ in Table A5, i.e., values for which the transcendental part of the desired sum vanishes, so that the answer agrees with the polynomial $-P(x)$. However, the actual function constructed by the $u$ transform is always a rational function, never just a 
polynomial. None of the other polynomials $Q(x)$ have any positive rational roots, and no other cases were found in which the accelerators could find even one correct digit of the desired answer when $x$ was less than about 20 .

Department of Mathematics

Duke University

Durham, North Carolina 27706

Computer Services Division

NASA Lewis Research Center

Cleveland, Ohio 44135

1. M. Abramowitz \&. I. A. Stegun (eds.), Handbook of Mathematical Functions with Formulas, Graphs, and Mathematical Tables, Nat. Bur. Standards Appl. Math. Series No. 55, Superintendent of Documents, U. S. Government Printing Office, Washington, D. C., 1964.

2. R. Bellman \&. R. KalABA, "A note on nonlinear summability techniques in invariant imbedding," J. Math. Anal. Appl., v. 6, 1963, pp. 465-472.

3. C. M. Bender \&. T. T. Wu, "Anharmonic oscillator," Phys. Rev., v. 184, 1969, pp. 1231-1260.

4. W. G. Bickley \&. J. C. P. MilleR, "The numerical summation of slowly convergent series of positive terms," Philos. Mag., 7th Ser., v. 22, 1936, pp. 754-767.

5. E. BoDEWIG, "A practical refutation of the iteration method for the algebraic eigenvalue problem," MTAC, v. 8, 1954, pp. 237-240.

6. C. BreZinsKi, "Accélération de suites à convergence logarithmique," C.R. Acad. Sci.Paris Sér. A-B, v. 273, 1971, pp. A727-A730.

7. C. BREZINSKI, "Computation of the eigenelements of a matrix by the $\varepsilon$-algorithm," Linear Algebra Appl., v. 11, 1975, pp. 7-20.

8. E. GeKelER, "On the solution of systems of equations by the epsilon algorithm of Wynn," Math. Comp., v. 26, 1972, pp. 427-436.

9. S. Graffi, V. Grecchi \&. B. Simon, "Borel summability: Application to the anharmonic oscillator," Phys. Lett. B, v. 32, 1970, pp. 631-634.

10. R. T. Gregory \&. D. L. Karney, A Collection of Matrices for Testing Computational Algorithms, Wiley-Interscience, New York, 1969.

11. P. HeNRICI, Elements of Numerical Analysis, Wiley, New York, 1964.

12. K. IGUCHI, "On the Aitken's $\delta^{2}$-process," Inform. Process. in Japan, v. 15, 1975, pp. 36-40.

13. K. IGUCHI, "An algorithm of an acceleration process covering the Aitken's $\delta^{2}$-process," Inform. Process. in Japan, v. 16, 1976, pp. 89-93.

14. D. LEVIN, "Development of non-linear transformations for improving convergence of sequences," Internat. J. Comput. Math., v. 3, 1973, pp. 371-388.

15. R. S. Martin, C. Reinsch \&. J. H. Wilkinson, "Householder's tridiagonalization of a symmetric matrix," Numer. Math., v. 11, 1968, pp. 181-195.

16. A. Ralston, A First Course in Numerical Analysis, McGraw-Hill, New York, 1965.

17. D. SHANKs, "Non-linear transformations of divergent and slowly convergent sequences," J. Math. and Phys., v. 34, 1955, pp. 1-42.

18. B. Simon, "Coupling constant analyticity for the anharmonic oscillator," Ann. Physics, v. 58, 1970, pp. $76-136$.

19. D. A. SMITH \&. W. F. Ford, "Acceleration of linear and logarithmic convergence," SIAM J. Numer. Anal., v. 16, 1979, pp. 223-240.

20. M. VAN DYKe, "Analysis and improvement of perturbation series," Quart. J. Mech. Appl. Math., v. 27,1974 , pp. $423-450$.

21. J. H. Wilkinson, "The use of iterative methods for finding the latent roots and vectors of matrices," MTAC, v. 9, 1955, pp. 184-191.

22. J. WIMP, "Toeplitz arrays, linear sequence transformations, and orthogonal polynomials," Numer. Math., v. 23, 1974, pp. 1-17.

23. P. WYNN, "On a device for computing the $e_{m}\left(S_{n}\right)$ transformation," MTAC, v. 10, 1956, pp. 91-96. 